\title{
Comment on Wang et al.: The efficacy of bevacizumab plus paclitaxel as first-line treatment for HER2-negative metastatic breast cancer: a meta-analysis of randomized controlled trials
}

\author{
Jun Li • Yunfeng Yao • Juehua Jing
}

Received: 23 March 2014 / Accepted: 1 April 2014 / Published online: 9 April 2014

(C) International Society of Oncology and BioMarkers (ISOBM) 2014

\section{Dear Editor,}

Recently, we carefully read the article by Wang et al. entitled "The efficacy of bevacizumab plus paclitaxel as first-line treatment for HER2-negative metastatic breast cancer: a meta-analysis of randomized controlled trials" published online in Tumor Biology [1]. The authors performed a well meta-analysis to evaluate the efficacy of bevacizumab combined with paclitaxel in HER2-negative metastatic breast cancer patients. Nevertheless, the question being asked is an important one; we have several worthwhile queries that we would like to communicate with the authors.

1. The authors only searched the literature from PubMed and EMBASE up to September 2013. In our experience, a more comprehensive search should include MEDLINE, SCOPUS, Science Citation Index, Cochrane-controlled Trials Register, the Cochrane Library, Chinese Biomedical Literature Database, Wanfang Database, China National Knowledge Infrastructure, and certain specialty database. Therefore, more electronic databases should be systematically searched. Meanwhile, the authors did not describe clearly the search strategy, which plays an important role in systematic reviews.

2. Manual searches were not clearly stated. The lack of a manual search protocol may be considered a drawback of this meta-analysis. We suggest that the authors provide a clear manual search protocol.

3. Publication language in this meta-analysis was limited to English, then a lot of non-English trials may have been missed, so language bias may be unavoidable. And the authors did not mention it in the discussion section; we

J. Li $\cdot$ Y. Yao $\cdot$ J. Jing $(\bowtie)$

Department of Orthopaedics, The Second Hospital, Anhui Medical

University, 230601 Hefei, Anhui, People's Republic of China

e-mail: jingjuehuapaper@163.com suggest that there be no language limitation which will reduce the bias.

4. "We included all the clinical trials that evaluated the administration of bevacizumab plus paclitaxel versus other chemotherapy in HER2-negative metastatic breast cancer. Eligibility articles also should comprise one of the following outcomes: overall survival, progression-free survival, and objective response rate," this inclusion criteria was not enough in the meta-analysis, and the exclusion criteria almost was not mentioned in the article. We suggest that strict inclusion and exclusion criteria should be described clearly.

5. The publication bias in this meta-analysis was assessed by Egger's and Begg's tests. However, the number of studies was only eight, as far as we know; a funnel plot should be inspected visually to assess for publication bias, in metaanalyses with at least ten studies. What is more, there were 13 points in the figure 2, but only eight trials were included; we hope that the authors could explain that.

Above all, we respect the contribution of the authors. The meta-analysis confirms the benefits of bevacizumab-paclitaxel combination therapy in HER2-negative metastatic breast cancer, with an improvement in both progression-free survival and objective response rate. We believe that our remarks will contribute to a more accurate elaboration of the results presented by Wang et al.

Conflicts of interest None

\section{Reference}

1. Wang X, Huang C, Li M, Gu Y, Cui Y, Li Y. The efficacy of bevacizumab plus paclitaxel as first-line treatment for HER2-negative metastatic breast cancer: a meta-analysis of randomized controlled trials. Tumour Biol. 2014. doi:10.1007/s13277-014-1635-4. 